\title{
Locally Advanced Perforated Appendiceal Cancer: Case Report and Review
}

\author{
Samik Patel ${ }^{1}$, John Eggenberger ${ }^{1}$, Beth-Ann Shanker ${ }^{1}$, and Robert Cleary ${ }^{1}$ \\ ${ }^{1}$ Affiliation not available
}

February 22, 2021

\begin{abstract}
Appendiceal cancers may be difficult to diagnose even after comprehensive investigation. This report of locally advanced perforated appendiceal adenocarcinoma attached to the terminal ileum, cecum, and rectosigmoid illustrates the management challenges that require comprehensive knowledge of pathologic variations and range from simple appendectomy to cytoreductive surgery and hyperthermic intraperitoneal chemotherapy.
\end{abstract}

\section{Introduction}

The incidence of appendiceal neoplasms is 1.2 cases per 100,000 persons per year in the United States and $1-2 \%$ of appendectomies. ${ }^{1}$ Management of appendiceal neoplasms is challenging partly due to classification complexity. Following SCARE criteria ${ }^{2}$, we present a patient with locally advanced and perforated appendiceal adenocarcinoma with no signs of peritoneal, solid organ or nodal metastases and without evidence of pseudomyxoma peritonei. To address pathologic variations and treatment algorithm inconsistencies, we also review epithelial appendiceal neoplasms based on the World Health Organization classification, current nomenclature, clinical presentations, and provide a proposed algorithm for management.

\section{Case Report}

A 58-year-old healthy man with no family history of colorectal cancer or inflammatory bowel disease presented to our colorectal clinic with a 6-month history of bilateral lower abdominal pain radiating to the back, 5-pound weight loss, and irregular watery bowel movements. He has one alcoholic drink per day and denied tobacco and recreational drug use. There was no nausea, vomiting, melena, hematochezia, shortness of breath, chest pain, dysuria or previous abdominal surgeries. On presentation his vitals were normal. Physical exam revealed no abdominal tenderness, distention, or palpable masses.DiagnosisPrior to the colorectal surgery clinic visit, a stool DNA test was ordered by his primary care physician and was negative. Laboratory studies were remarkable only for a carcinoembryonic antigen (CEA) level of $5.9 \mathrm{ng} / \mathrm{mL}$ He was initially diagnosed with irritable bowel syndrome. Colonoscopy performed by the gastroenterologist revealed a long rectosigmoid stricture that required a gastroscope to negotiate. The cecum and appendiceal orifice appeared normal. Biopsies of the rectosigmoid stenosis showed no significant pathologic abnormality and was non-diagnostic. Computed tomography (CT) imaging of the abdomen and pelvis demonstrated an irregular rim-enhancing heterogeneous mass measuring $6.7 \mathrm{~cm} \mathrm{x} 2.8 \mathrm{~cm}$, originating from the base of the cecum and extending across the midline and tethered to the rectosigmoid junction without evidence of fistula (Figure 1). CT-guided fine needle aspiration was unsuccessful. The patient was then referred to the colorectal 
surgery clinic for further evaluation.Differential DiagnosisConsidering patient symptoms, CT imaging, and endoscopic findings, the differential diagnosis included possible colorectal malignancy, perforated appendix with pelvic abscess formation with involvement of the rectosigmoid junction, perforated colon cancer with abscess formation, or inflammatory bowel disease with fistula and abscess formation. Treatment The patient underwent an exploratory laparotomy for concern of possible malignancy and near- obstructing rectosigmoid stricture. An abscess cavity involving the terminal ileum, distal sigmoid and proximal rectum was identified. Mobilization of the right colon revealed a pocket of purulent material posterior to the proximal ascending colon. En-bloc resection of the terminal ileum, right colon, sigmoid and proximal rectum was performed with two primary anastomoses (ileocolic and colorectal) without stomas. OutcomeIntraoperative pelvic fluid cultures grew Streptococcus viridans and the patient was treated with appropriate antibiotics. Pathology showed perforated appendiceal moderately differentiated adenocarcinoma with abscess formation and direct invasion of the rectum without lymph node involvement (Stage IIC T4bN0Mx, 0/34 lymph nodes). Postoperative course was unremarkable and the patient was discharged home without complications and meeting discharge criteria. The patient was presented at the multidisciplinary tumor board and 6-12 cycles of systemic FOLFOX chemotherapy was recommended because of high-risk features that included T4 depth of invasion, perforation and adherence to the rectosigmoid, concern for micrometastasis, and lymphovascular invasion. However, the patient refused chemotherapy and instead opted for routine surveillance with CEA levels and CT imaging. Postoperative CEA level one month after surgery was 1.7 (from 5.9 and currently 4.3). CT of the chest/abdomen/pelvis 6 months after surgery showed no evidence of recurrence or metastasis. The patient is now 12 months since surgery without evidence of recurrent disease and training for a half marathon.

\section{Discussion}

The World Health Organization classification scheme divides appendiceal neoplasms into epithelial tumors, mesenchymal tumors, lymphomas, and secondary tumors. The epithelial tumors include premalignant lesions (adenomas and serrated lesions), carcinomas (mucinous adenocarcinoma, low-grade appendiceal mucinous neoplasm (LAMN), signet ring cell carcinoma, undifferentiated carcinoma) and neuroendocrine neoplasms. ${ }^{2,3}$ The diagnosis of appendiceal neoplasm may be challenging and requires thorough history and physical examination, laboratory studies, imaging (CT/ MRI), endoscopy and diagnostic tumor biopsy or surgery. Serum carcinoembryonic antigen (CEA) levels should be obtained as for colorectal cancers. Colonoscopy may show an appendiceal orifice mass. Tumor markers CA-125 and CA 19-9 may be elevated in patients with appendiceal primary tumors and peritoneal disease. ${ }^{4}$ Normal levels of CA-125 and CA 19-9 are associated with improved survival and decreased rates of recurrence. ${ }^{5} \mathrm{CT}$ of the chest, abdomen, and pelvis allows evaluation of the primary tumor and assessment of metastatic disease. ${ }^{5}$ The diagnosis is often unknown prior to operative intervention for presumed appendicitis. Malignant neoplasia is identified in 2.3-12.0\% of patients having appendectomy for appendicitis. Risk factors for appendiceal cancer in this appendicitis group are older age and periappendiceal abscess. ${ }^{2}$ Risk factors that predispose locally invasive appendiceal adenocarcinoma to intraperitoneal dissemination and metastasis have been described. ${ }^{6}$ One study showed that T4 depth of invasion, N2 nodal status, and mucinous tumor were associated with peritoneal metastasis. ${ }^{6}$ Another study showed that the incidence of lymph node metastasis was associated with both larger tumor size and advanced T stage. ${ }^{7}$ The patient described in this case report had a T4b neoplasm but had no lymph node metastases and has no evidence of metastatic disease to date. Management of appendiceal neoplasms varies depending on the pathology. For appendiceal adenocarcinoma, treatment depends on whether the neoplasm has perforated and led to intraperitoneal dissemination. There is extensive literature describing ruptured appendiceal adenocarcinoma with widespread intra-peritoneal mucinous ascites leading to pseudomyxoma peritonei (PMP) and peritoneal carcinomatosis. However, there are currently no standard guidelines for management of locally invasive perforated appendiceal adenocarcinoma without PMP or intra-peritoneal dissemination. ${ }^{2,3}$ For patients like the one presented in this case report with locally advanced perforated appendiceal adenocarcinoma without PMP or carcinomatous, en-bloc R0 resec- 
tion, when possible, with postoperative systemic chemotherapy would likely be the best option. The lack of standardized nomenclature for appendiceal neoplasms contributes to treatment algorithm inconsistencies. The following is the most recent update in pathologic terminology and management recommendations for the most common appendiceal neoplasms. The proposed algorithm for management is shown in Figure 2. Appendiceal AdenomaAppendiceal adenomas are benign polyps of the mucosa like adenomas elsewhere in the colon and rectum. Most do not have presenting symptoms and are found incidentally but some patients may have symptoms of appendicitis. ${ }^{3}$ Histologic exam resembles colonic tubular, villous, or tubulovillous adenomas. There is cellular crowding and often low-grade dysplastic changes of nuclear elongation and stratification, pleomorphism, and increased mitotic figures. Adenomas are associated with KRAS mutations and loss of chromosome 5q. The majority of adenomas are microsatellite stable and lack BRAF mutations. Like colonic adenomas, appendiceal adenomas are premalignant and follow the adenoma-carcinoma sequence. ${ }^{8}$ Treatment for appendiceal adenoma confined to the mucosa is appendectomy with negative margins.Low-Grade Appendiceal Mucinous NeoplasmPatients with low-grade appendiceal mucinous neoplasms (LAMN) range in age from 20-90 years with most in the $7^{\text {th }}$ decade. They are more common in women. ${ }^{3}$ Patients present with symptoms of appendicitis or abdominal pain, distension or an abdominal mass. LAMNs have uncertain malignant potential. ${ }^{2}$ About 15-20\% are found incidentally when undergoing surgery for an unrelated etiology. ${ }^{3}$ Grossly, the appendix appears normal but may be dilated due to intraluminal mucin accumulation. They may also present with appendiceal perforation, mucin within the appendiceal wall, or acellular mucin outside of the appendix. ${ }^{2}$ On histologic exam, LAMNs consist of well differentiated, glandular, mucinous neoplastic epithelial cells. ${ }^{1,3}$ LAMNs frequently have KRAS mutations and loss of chromosome $5 \mathrm{q}^{3}{ }^{3}$ Almost $100 \%$ of mucinous appendiceal neoplasms express Keratin 20, CDX2 and MUC2. ${ }^{3}$ They are also associated with GNAS mutations with elevated cAMP levels leading to increased expression of MUC2 and MUC5AC and increased mucin production. ${ }^{3}$ Prognosis depends on LAMN perforation and the presence of mucinous neoplastic epithelium outside the appendix. LAMNs confined to the appendix with no extra-appendiceal perforation are treated by appendectomy with negative margins. This may require a partial cecectomy if the tumor involves the base of the appendix. ${ }^{2,5}$ The specimen should be placed in a retrieval bag when done by the laparoscopic approach to decrease the risk for mucin spillage. When the risk for mucin spillage is considered high, an open approach should be considered. LAMNs with extra-appendiceal acellular mucin have a low 3-7\% risk for peritoneal recurrence and generally have a good prognosis. LAMNs with intraperitoneal spread of cellular mucin containing neoplastic epithelium have a higher $33-78 \%$ risk of peritoneal recurrence and overall poorer prognosis. ${ }^{3}$ Patients with perforated LAMNs with intraperitoneal spread of acellularmucin should have routine surveillance imaging to rule out peritoneal recurrence or disease progression. ${ }^{2,3} \mathrm{~Pa}-$ tients with perforated LAMNs with intraperitoneal cellular mucin containing neoplastic epithelium should undergo a right hemicolectomy or right hemicolectomy with cytoreductive surgery (CRS) and hyperthermic intraperitoneal chemotherapy (HIPEC) ${ }^{2,3}$ High-grade appendiceal neoplasms are histologically similar to LAMNs but have more aggressive cytologic atypia. ${ }^{2}$ Adenocarcinoma Appendiceal adenocarcinomas occur in the $5^{\text {th }}-7^{\text {th }}$ decade with mean age 60 and are more common in men. ${ }^{1}$ Patients may present with signs and symptoms of acute appendicitis. Adenocarcinomas may be mucinous or nonmucinous and are subclassified into adenocarcinoma not otherwise specified, mucinous adenocarcinoma, signet ring cell adenocarcinoma and undifferentiated carcinoma. ${ }^{3}$ Mucinous adenocarcinoma is the most common adenocarcinoma of the appendix - about $40 \%$ of all adenocarcinomas. They consist of malignant glandular mucinous epithelium within the wall of the appendix with infiltrative destructive invasion, high- grade cytologic atypia and extracellular mucin in $>50 \%$ of the neoplasm. ${ }^{2,3}$ Adenocarcinomas are associated with mutations of chromosome $18 \mathrm{q}$, frequently have KRAS mutations, and histologically express p53, CD44, and CDX2. ${ }^{3}$ Intraperitoneal dissemination of mucinous adenocarcinoma is similar to LAMN except that the malignant epithelium contains high-grade cytologic atypia and the mucin is more cellular. ${ }^{3}$ Signet ring cell adenocarcinomas are rare and have a poor prognosis. ${ }^{5}$ Non-mucinous adenocarcinomas, like that presented in this case report, behave like colonic adenocarcinomas. Patients with invasive mucinous or nonmucinous adenocarcinomas and signet ring cell adenocarcinomas should undergo a right hemicolectomy with lymphadenectomy due to 20-67\% risk of lymph node metastases. ${ }^{1}$ These patients require oncologic resection that includes high ligation of mesenteric vessels, removal of the associated lymph node basin, and grossly negative 5-cm margins. Patients 
with mucinous adenocarcinoma with widespread intraperitoneal deposits should undergo CRS and HIPEC. There is no advantage to right hemicolectomy over appendectomy when peritoneal metastases are present unless it is necessary to achieve complete cytoreduction. ${ }^{2,3}$ Systemic chemotherapy should be administered to patients with lymph node or distant organ metastases. Goblet cell carcinoid tumors are mixed adenoneuroendocrine carcinomas with neuroendocrine tumor features such as positive chromogranin A staining. They are clinically more aggressive than neuroendocrine tumors and treatment is the same as for appendiceal adenocarcinomas. ${ }^{2}$ Pseudomyxoma PeritoneiPMP is characterized by appendiceal neoplasm perforation with intraperitoneal accumulation of mucin resulting in gelatinous mucinous ascites. ${ }^{5,9} \mathrm{PMP}$ occurs in $20 \%$ of patients with mucinous appendiceal neoplasms and more commonly in females (ratio 4:1) between the ages of 60-70. ${ }^{9}$ In 2010, the American Joint Committee on Cancer (AJCC) and World Health Organization (WHO) classified PMP into 2 groups: low-grade PMP and high-grade PMP. ${ }^{5,8,9}$ Low-grade PMP, also known as diffuse peritoneal adenomucinosis (DPAM), is associated with LAMN and is characterized by intraperitoneal spread of acellular or cellular mucin with low-grade cytology and no significant mitotic activity. ${ }^{8} 9$ The mucin in the peritoneum adheres to but does not invade or penetrate surrounding visceral structures within the peritoneum. ${ }^{5}$ Normal baseline CA-125 and CEA returning to normal after surgery are associated with the ability to achieve complete cytoreduction in this patient population. Elevated baseline CA19-9 is associated with worse progression-free survival. ${ }^{2}$ High-grade PMP, also known as peritoneal mucinous carcinomatosis (PMCA), is associated with mucinous adenocarcinoma and is characterized by intraperitoneal spread of mucin containing high-grade malignant neoplastic epithelium with moderate to severe cytologic atypia..$^{8,9}$ High-grade PMP has a strong predilection for peritoneal lesions that invade visceral structures. ${ }^{5}$ There is considerable debate over the management of PMP and the treatment algorithm has not been standardized. Prior to CRS, treatment was surgical debulking. Sugarbaker introduced and popularized the use of CRS and HIPEC for patients with PMP. ${ }^{5,9}$ CRS removes all visible macroscopic disease and HIPEC is used to provide directed highly concentrated intraperitoneal chemotherapy to penetrate any remaining microscopic disease. ${ }^{5}$ Right hemicolectomy with cytoreductive surgery (CRS) and hyperthermic intraperitoneal chemotherapy (HIPEC) is currently recommended for appendiceal adenocarcinoma with peritoneal carcinomatosis or PMP. HIPEC most commonly includes Mitomycin C or platinum chemotherapy agents. ${ }^{2}$ Several studies have shown improved prognosis and disease-free survival in patients having CRS and HIPEC for appendiceal cancer associated with peritoneal carcinomatosis. ${ }^{10,11}$ Furthermore, patients who underwent both CRS and HIPEC versus CRS alone had improved survivability. ${ }^{10}$ Five- and ten-year survival rates for low-grade PMP are $75 \%$ and $68 \%$ respectively. Five- and ten-year survival rates for high-grade PMP are $14 \%$ and $3 \%$ respectively. Several studies have shown no clear disease-free survival or overall survival advantage for patients who underwent preoperative chemotherapy prior to CRS and HIPEC. ${ }^{12}$ The use of early postoperative intraperitoneal chemotherapy (EPIC) has been shown to improve survival in patients with appendiceal adenocarcinoma with peritoneal carcinomatosis compared with HIPEC alone without increasing postoperative morbidity and mortality. ${ }^{4} 13$ Patients who undergo CRS and HIPEC require routine surveillance for recurrence that includes physical examination, CT imaging of the chest, abdomen and pelvis and tumor markers (CEA, CA-125, CA 19-9) every 6 months for the first 2 years, and then yearly for at least 3 years. MRI may be superior to CT for postoperative surveillance after (CRS) and (HIPEC). ${ }^{5}$ Serrated Tumors of the AppendixSerrated tumors of the appendix occur in older patients and affect both males and females equally. ${ }^{3}$ They are found incidentally or in patients who present with signs and symptoms of appendicitis. They are classified into hyperplastic polyps, sessile serrated adenoma/polyps $(+/-$ cytologic dysplasia), and traditional serrated adenoma/polyps (+/- cytologic dysplasia). ${ }^{11}$ Hyperplastic polyps are benign and have low risk for malignant transformation. Sessile serrated polyps consist of serrated, dysplastic epithelium in the basal crypts with increased mitotic figures and mild nuclear atypia. ${ }^{3}$ Traditional serrated adenomas have villous growth pattern with complex serration. Due to the risk of malignant transformation, serrated neoplasms confined to the appendix without involvement of the adjacent cecum are treated by appendectomy with negative margins. Right hemicolectomy with regional lymphadenectomy is the preferred option for patients with large serrated lesions suspicious for malignant degeneration or those that involve the cecum or other organs. This case report adds to current literature by providing an unusual presentation of appendiceal adenocarcinoma, by providing a review of classification schemes and pathologic variations, and 
by providing a proposed treatment algorithm.

\section{Legends (All Figures will need to be in color for print)}

Figure 1. CT imaging of irregular pelvic mass arising from cecum with extension to the rectosigmoid junction Figure 2. Classification and Management of Appendix Epithelial Neoplasms

\section{Data Availability}

All of the data is included in this report.

\section{Conflict of Interest}

Samik H. Patel MD has no conflict of interest to report. John C. Eggenberger MD has no conflict of interest to report. Beth-Ann Shanker MD has no conflict of interest to report. Robert K. Cleary has no conflict of interest to report related to this research. He has received honoraria from Intuitive Surgical, Inc. for educational speaking.

\section{Authorship}

Samik H Patel MD: Substantial contributions to the design of the work and analysis and interpretation of data for the work; Drafting the work or revising it critically for important intellectual content; Final approval of the version to be published; Agreement to be accountable for all aspects of the work in ensuring that questions related to the accuracy or integrity of any part of the work are appropriately investigated and resolved.

John C Eggenberger MD: Substantial contributions to the design of the work; Drafting the work or revising it critically for important intellectual content; Final approval of the version to be published; Agreement to be accountable for all aspects of the work in ensuring that questions related to the accuracy or integrity of any part of the work are appropriately investigated and resolved.

Beth-Ann Shanker MD: Substantial contributions to the design of the work; Drafting the work or revising it critically for important intellectual content; Final approval of the version to be published; Agreement to be accountable for all aspects of the work in ensuring that questions related to the accuracy or integrity of any part of the work are appropriately investigated and resolved.

Robert K Cleary MD: Substantial contributions to the design of the work and analysis and interpretation of data for the work; Drafting the work or revising it critically for important intellectual content; Final approval of the version to be published; Agreement to be accountable for all aspects of the work in ensuring that questions related to the accuracy or integrity of any part of the work are appropriately investigated and resolved.

\section{Funding Statement}

No funding was received for this research.AcknowledgmentsThere are no other acknowledgments.

\section{References}

1. Kelly KJ. Management of Appendix Cancer. Clin Colon Rectal Surg . 2015;28(4):247-255. 
2. Glasgow SC, Gaertner W, Stewart D, Davids J, Alavi K, Paquette IM, Steele SR, Feingold DL. The American Society of Colon and Rectal Surgeons, Clinical Practice Guidelines for the Management of Appendiceal Neoplasms. Dis Colon Rectum 2019;62:1425-1438

3. Odze RD, Goldblum JR. Epithelial Neoplasms of the Appendix. In: Odze and Goldblum Surgical Pathology of the GI Tract, Liver, Biliary Tract and Pancreas. Edition 3. Elsevier Health Services; 2014;779-802

4. Huang Y, Alzahrani NA, Liauw W, et al. Early Postoperative Intraperitoneal Chemotherapy for LowGrade Appendiceal Mucinous Neoplasms with Pseudomyxoma Peritonei: Is it Beneficial? Ann Surg Oncol . 2017 Jan;24(1):176-183.

5. Lansom J, Alzahrani N, Liauw W, et al. Cytoreductive Surgery and Hyperthermic Intraperitoneal Chemotherapy for Pseudomyxoma Peritonei and Appendix Tumours. Indian J Surg Oncol . 2015;7(2):166-176.

6. Enblad M, Graf W, Birgisson H. Risk factors for appendiceal and colorectal peritoneal metastases. Eur J Surg Oncol . 2018 Jul;44(7):997-1005.

7. Gahagan JV, Whealon MD, Phelan MJ, et al. Lymph Node Positivity in Appendiceal Adenocarcinoma: Should Size Matter? J Am Coll Surg . 2017 Jul;225(1):69-75.

8. Tirumani SH, Fraser-Hill M, Auer R, et al. Mucinous neoplasms of the appendix: a current comprehensive clinicopathologic and imaging review. Cancer Imaging . 2013;13(1):14-25.

9. Ramaswamy V. Pathology of Mucinous Appendiceal Tumors and Pseudomyxoma Peritonei.

Indian J Surg Oncol. 2016;7(2):258-267.

1. Votanopoulos KI, Russell G, Randle RW, et al. Peritoneal surface disease (PSD) from appendiceal cancer treated with cytoreductive surgery (CRS) and hyperthermic intraperitoneal chemotherapy (HIPEC): overview of 481 cases. Ann Surg Oncol . 2015 Apr;22(4):1274-9.

2. Gupta N, Asif S, Gandhi J, et al. Role of CRS and HIPEC in appendiceal and colorectal malignancies: Indian experience. Indian J Gastroenterol . 2017 Mar;36(2):126-130.

3. Cummins KA, Russell GB, Votanopoulos KI, et al. Peritoneal dissemination from high-grade appendiceal cancer treated with cytoreductive surgery (CRS) and hyperthermic intraperitoneal chemotherapy (HIPEC). J Gastrointest Oncol . 2016 Feb;7(1):3-9.

4. Huang Y, Alzahrani NA, Liauw W, et al. Early postoperative intraperitoneal chemotherapy is associated with survival benefit for appendiceal adenocarcinoma with peritoneal dissemination. Eur J Surg Oncol . 2017 Dec;43(12):2292-2298.

Figure 1. CT imaging of irregular pelvic mass arising from cecum with extension to the rectosigmoid junction
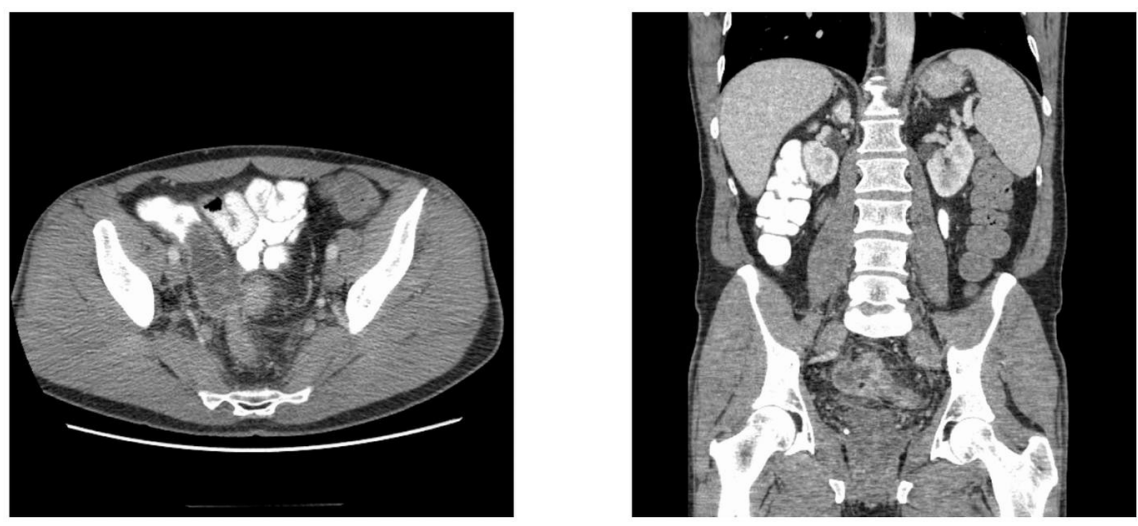

Figure 2. Classification and Management of Appendicular Epithelial Neoplasms 

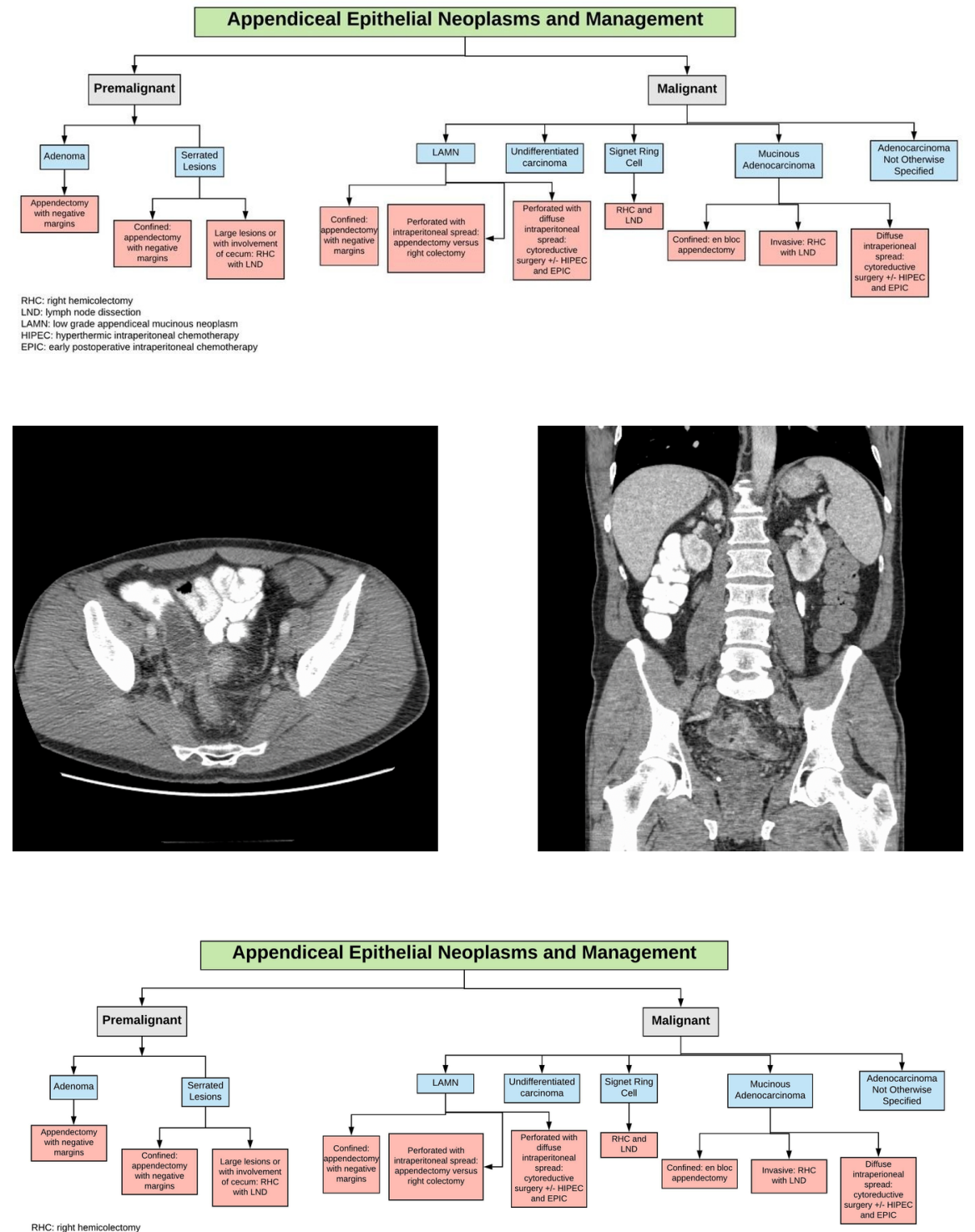

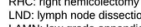

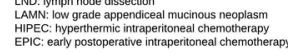

